\title{
Evaluation of the Time to Terminate Continuous Renal Replacement Therapy in Patients with Acute Kidney Injury Following Cardiovascular Surgery in Association with or without a Mechanical Ventilation Support
}

\author{
Isao Tsukamoto', Youhei Tsuchiya1, Hiromichi Suzuki ${ }^{2,3}$, Yusuke Watanabe ${ }^{2,3}$, Keita Sueyoshi²,3 and Hirokazu Okada²,3
}

${ }^{1}$ Division of Medical Engineering, Saitama Medical University, Japan

${ }^{2}$ Division of Blood purification, Saitama Medical University, Japan

${ }^{3}$ Department of Nephrology, International Medical Center, Saitama Medical University, Japan

\begin{abstract}
Acute kidney injury (AKI) is a frequent and severe complication after cardiovascular surgery. Indications for continuous renal replacement therapy (CRRT) in patients with AKI have been proposed. However, there is often less discussion of when to terminate CRRT as well as what conditions are required for transferring patients to intermittent hemodialysis (HD). In this retrospective study of electronic medical records, we examined the role of a mechanical ventilator support in determining when to terminate CRRT in patients with AKI. When CRRT was stopped, 32 patients were supported with a mechanical ventilator while 41 patients had no support. Although there were no differences in age, sex, and pre levels of cardiopulmonary between the two groups, the levels of eGFR before the cardiovascular surgery were lower in the patients without a ventilator. Increases in body weight after cardiac surgery were higher, sequential organ failure assessment (SOFA) score was higher, and central venous pressures were higher in patients supported with a ventilator (all were $p<0.05)$. The rate of re-introduction to CRRT was higher $(p=0.016)$ in patients supported with a ventilator. After multiple regression analysis, successful cessation of CRRT was dependent on support of mechanical ventilation (odds ratio, 5.20 (Cl: 1.15-23.4)). These data suggest that successful termination of CRRT is closely associated with removing the support of a mechanical ventilator.
\end{abstract}

\section{Keywords: SOFA score; Urine volume; Fluid therapy}

\section{Introduction}

The abrupt cessation of homeostasis induces multiple organ failure and cardiac surgery is one of the procedures that results in disturbance of homeostasis. Acute kidney injury (AKI) is a major postoperative complication in cardiac surgery, and is associated with high morbidity and mortality [1]. The reported incidence of cardiac surgeryassociated AKI based on its many historical definitions ranges from 0.3 to $29.7 \%$ [2,3]. In 1.2 to $3.0 \%$ of those patients, renal replacement therapy (RRT) is required $[4,5]$. There is a wide variation throughout the world on the indications for, and for when RRT is started [6-8]. Moreover, the termination of continuous RRT (CRRT) is affected by strongly held physician beliefs and organizational aspects of a given institution. Uchino et al. [9] were the first to report that the time of CRRT termination depended on urine output. In addition to low urine output, Wu et al. [10] proposed that older age, higher SOFA scores, and a longer dialysis-dependent period were risk factors for early redialysis. In general, patients suffering from AKI after cardiac surgery are treated in intensive care units (ICU). In this situation, all patients are usually intubated and their respiratory function is supported by a mechanical ventilator. Although the effective clinical protocols for the prevention and optimal management for AKI after cardiac surgery are not yet established, generally proposed strategies for its prevention stress optimal support of cardiovascular function and oxygen delivery during and after surgery. For mechanical ventilation, there are several international consensus guidelines that have been proposed, which have been followed by physicians working in ICU [11]. In our practice, the two on renal and respiratory mechanical support might be considered together. In other words, it might be appropriate that weaning from mechanical ventilation and cessation of CRRT are simultaneously carried out for acutely ill patients after cardiac surgery. In the present study, we examined the association of weaning from a mechanical ventilator and its cessation in AKI patients requiring continuous CRRT after cardiac surgery.

\section{Subjects and Methods}

This retrospective observational study was performed between July 2008 and September 2012 in a cohort of adult patients who underwent open heart surgery and needed CRRT due to AKI in the International Medical Center Saitama Medical University, Saitama, Japan. This study was approved by the institutional review board of our institution. The inclusion criteria were AKI patients who started CRRT while receiving respiratory support of a mechanical ventilator after cardiac surgery and those who stopped CRRT with or without a mechanical ventilator. The patients were divided into 2 groups: A) patients who were supported by a mechanical ventilator when CRRT was terminated and B) patients who were not supported by a mechanical ventilator when CRRT was terminated. Weaning of mechanical ventilation was decided by the intensivists who followed the guidelines. The cessation of CRRT was determined by integrated assessment of increased urine output, use of vasopressors, improved metabolic/electrolyte state, decreased urea/creatinine, and stable hemodynamics. A successful weaning was defined as the cessation of dialysis for at least 30 days.

AKI was defined according to the Second International Consensus Conference of the Acute Dialysis Quality Initiative Group with

*Corresponding author: Hiromichi Suzuki, Department of Nephrology, Saitama Medical University, Saitama, Japan; Tel: +81-49276-1620; E-mail: iromichi@saitama-med.ac.jp

Received July 17, 2014; Accepted November 14, 2014; Published November 20, 2014

Citation: Tsukamoto I, Tsuchiya Y, Suzuki H, Watanabe $\mathrm{Y}$, Sueyoshi K, et al (2014) Evaluation of the Time to Terminate Continuous Renal Replacement Therapy in Patients with Acute Kidney Injury Following Cardiovascular Surgery in Association with or without a Mechanical Ventilation Support. J Nephrol Ther 5: 188. doi:10.4172/2161-0959.1000188

Copyright: (c) 2014 Tsukamoto I, et al. This is an open-access article distributed under the terms of the Creative Commons Attribution License, which permits unrestricted use, distribution, and reproduction in any medium, provided the original author and source are credited. 
Citation: Tsukamoto I, Tsuchiya Y, Suzuki H, Watanabe Y, Sueyoshi K, et al. (2014) Evaluation of the Time to Terminate Continuous Renal Replacement Therapy in Patients with Acute Kidney Injury Following Cardiovascular Surgery in Association with or without a Mechanical Ventilation Support. J Nephrol Ther 5: 188. doi:10.4172/2161-0959.1000188

Page 2 of 4

reference to RIFLE (risk, injury, failure, loss, and end-stage kidney disease) [12]. Continuous venovenous hemofiltration (CRRT) was started when at least one of the following institutional protocol criteria was fulfilled: (i) urine output below $0.5 \mathrm{~mL} / \mathrm{kg} / \mathrm{h}$ in 6 hours despite treatment with fluid transfusion, inotropes, and/or vasoconstrictor infusions aimed at optimization of the hemodynamic parameters and the administration of furosemide $100 \mathrm{mg} / \mathrm{h}$ over 3 hours or (ii) more than a 4 -fold increase in plasma creatinine concentration was observed. Hemofiltration treatment ceased when patients recovered urine output exceeding $1 \mathrm{~mL} / \mathrm{kg} / \mathrm{h}$, provided that no indications for RRT were observed subsequently. Patients were accessed through double-lumen catheters (Vas-cath, Medicon Co., Chicago, IL), which were inserted into the right or left femoral vein and connected to a continuous hemodialyzer (KM8600, Kurary Co. Ltd., Tokyo, Japan). An anticoagulant, nafamostat mesilate (Futhan, Torii Pharmaceutical Co. Ltd. Tokyo, Japan), was used at $30 \mathrm{IU} / \mathrm{hr}$. Dialysis was started under conditions of water elimination rate of $60 \mathrm{~mL} / \mathrm{hr}$ and a dialysate (HF Solita, Shimizu Pharmaceutical Co., Ltd., Shimizu, Japan) flow rate of $1 \mathrm{~L} / \mathrm{hr}$. The dialyzers used in this study were Panflow APF-S (Asahi Medical Co., Ltd., Tokyo, Japan) and Hemofeel SH (Toray Medical Co., Ltd., Tokyo, Japan).

Disease severity was assessed using the Sequential Organ Failure Assessment (SOFA) score [13].

eGFR was calculated using a modified three-variable equation for eGFR in Japanese patients: eGFR $=194 \mathrm{xage}^{-0.287} \mathrm{x} \mathrm{SCr}^{-1.094}(\mathrm{x} 0.739$, if female), where SCr=serum creatinine [14]. Early redialysis, defined as relapsed need for CRRT within 30 days after cessation of CRRT, was considered the primary outcome variable. The indications for resumption of redialysis were mainly fluid overload and azotemia or its combination. The characteristics of patients were examined with regard to presence or absence of a mechanical ventilator for values of baseline, preoperative, and at cessation of CRRT.

\section{Statistical Analysis}

Data were analyzed by using SAS 1999 program (release 8.00 by SAS Institute Inc, Cary, NC). Continuous measures are expressed as mean \pm standard deviation and were compared using Student's $t$ test. Categorical variables were compared with Fisher' exact $\chi^{2}$ test. A multivariate logistic regression analysis with successful cessation of CRRT was carried out as dependent variables (age, preoperative eGFR, organ damage score at the cessation of CRRT, body weight changes, CVP and duration of CRRT). $\mathrm{P}<0.05$ was considered to be statistically significant.

\section{Results}

\section{Patient characteristics}

The demographics of patients in groups A and B are shown in (Table 1). There were no differences between the two groups in age, sex ratio, body weight, HbA1C, ejection fraction, presence of emergency surgery, types of cardiac surgery, duration of surgery, and use of IABP between the two groups. The only difference was eGFR of patients in group $\mathrm{B}$ was lower than in group $A(p<0.001)$. Moreover, at the initiation of CRRT, there were no differences in increases in serum creatinine and body weight, decreasing urine volume, central venous pressure and SOFA score at the initiation of CRRT between the two groups, (Table 2) shows variables at cessation of CRRT. In spite of elevations of serum creatinine in the two groups, group A showed higher urine volume
$(\mathrm{P}=0.016)$, higher dose of fluid therapy $(\mathrm{P}<0.001)$, higher body weights $(\mathrm{P}=0.032)$, higher CVP $(\mathrm{P}=0.003)$ and greater increases of SOFA scores (cardiovascular: $\mathrm{P}<0.001$; respiratory: $\mathrm{P}<0.001$; and liver: $\mathrm{P}=0.005$ ) than in patients of group $B$. On the contrary, renal scores of patients in group B were higher than in group A. In the analysis for SOFA score, neurological score was not counted, because "1" was given for patients of group A. Consequently, SOFA scores in group A were significantly higher than in group $B(10.7 \pm 2.2$ vs. $7.6 \pm 2.7 ; \mathrm{P}<0.01)$.

\begin{tabular}{|c|c|c|c|}
\hline & Group A(n=32) & Group B(n=41) & p-value \\
\hline Age (yr) & $72.5 \pm 6.4$ & $75.5 \pm 6.9$ & 0.062 \\
\hline Gender(M/F) & $19 / 13$ & $28 / 13$ & 0.468 \\
\hline Preoperative & & & \\
\hline Body weight(Kg) & $57.6 \pm 11.2$ & $57.4 \pm 12.7$ & 0.935 \\
\hline HbA1c(\%NGSP) & $6.23 \pm 1.17$ & $5.93 \pm 0.95$ & 0.193 \\
\hline Ejection Fraction (\%) & $48.5 \pm 16.2$ & $50.3 \pm 17.8$ & 0.655 \\
\hline eGFR(mL/min/1.73m $\left.{ }^{2}\right)$ & $46.1 \pm 19.6$ & $32.9 \pm 15.3$ & 0.001 \\
\hline Intraoperative & & & \\
\hline Emergency surgery(yes/no) & $10 / 22(31 \%)$ & $15 / 26(37 \%)$ & 0.804 \\
\hline Surgery & & & \\
\hline CABG & $19(59 \%)$ & $24(59 \%)$ & 1 \\
\hline Valve surgery & $8(25 \%)$ & $12(29 \%)$ & 0.793 \\
\hline CABG + valve & $5(16 \%)$ & $5(12 \%)$ & 0.739 \\
\hline Duration of surgery(hr) & $8.08 \pm 185$ & $7.51 \pm 1.87$ & 0.197 \\
\hline IABP(yes/no) & $17 / 15(53 \%)$ & $18 / 23(44 \%)$ & 0.484 \\
\hline At the start of CRRT & & & \\
\hline Increases in Scr (x) & $1.95 \pm 0.79$ & $1.65 \pm 0.58$ & 0.071 \\
\hline Urine volume(mL/hr/Kg) & $0.53 \pm 0.44$ & $0.67 \pm 0.49$ & 0.22 \\
\hline Changes in body weight (\%) & $9.08 \pm 5.41$ & $9.49 \pm 7.18$ & 0.788 \\
\hline CVP(mmHg) & $10.9 \pm 3.8$ & $9.8 \pm 3.9$ & 0.209 \\
\hline SOFA score & $12.2 \pm 2.4$ & $11.2 \pm 2.1$ & 0.084 \\
\hline
\end{tabular}

Values are expressed as mean \pm standard deviation. eGFR, estimated glomerula filtration rate; $\mathrm{CABG}$,

Coronary artery bypass grafting; IABP, intra-aortic balloon pump; Scr, serum creatinine;

CVP, central venous pressure; SOFA, sequential organ failure assessment.

Table 1: Demographic and clinical characteristics of acute kidney injury patients requiring continuous renal replacement therapy (CRRT) who had supported (Group A) or not supported (Group B) by mechanical ventilator at timing of cessation of CRRT.

\begin{tabular}{|c|c|c|c|}
\hline & Group A(n=32) & Group B (n=41) & p-value \\
\hline Increases in Scr (x) & $1.39 \pm 0.61$ & $1.19 \pm 0.34$ & 0.107 \\
\hline Urine volume(mL/day) & $2304 \pm 1372$ & $1608 \pm 1061$ & 0.016 \\
\hline Fluid therapy(mL/day) & $2457 \pm 667$ & $1938 \pm 472$ & $<.001$ \\
\hline Changes in body weight (\%) & $5.12 \pm 6.82$ & $2.94 \pm 6.65$ & 0.032 \\
\hline CVP(mmHg) & $6.6 \pm 3.4$ & $4.2 \pm 3.1$ & 0.003 \\
\hline SOFA score & $10.7 \pm 2.2$ & $7.6 \pm 2.7$ & $<.001$ \\
\hline Cardiovascular & $2.3 \pm 0.7$ & $1.5 \pm 0.8$ & $<.001$ \\
\hline Respiratory & $1.5 \pm 0.7$ & $1.1 \pm 0.8$ & 0.043 \\
\hline coagulation & $1.6 \pm 0.8$ & $1.3 \pm 1.0$ & 0.264 \\
\hline Liver & $1.9 \pm 1.3$ & $1.2 \pm 1.1$ & 0.005 \\
\hline Renal & $1.1 \pm 1.1$ & $1.7 \pm 1.2$ & 0.046 \\
\hline Organ damage & $8.5 \pm 2.1$ & $6.7 \pm 2.5$ & 0.001 \\
\hline Dose of dialysis(ml/hr/kg) & $9.6 \pm 4.3$ & $9.1 \pm 2.4$ & 0.549 \\
\hline Duration of CRRT(day) & $7.0 \pm 6.6$ & $7.0 \pm 3.8$ & 0.148 \\
\hline
\end{tabular}

Values are expressed as mean + standard deviation. Scr, serum creatinine; CVP central venous pressure; SOFA, sequential organ failure assessment; CRRT, continuous renal replacement therapy.

Table 2: Characteristics of patients with (group A) or without (group B) support of mechanical ventilation at the timing of cessation of continuous renal replacement therapy. 
Citation: Tsukamoto I, Tsuchiya Y, Suzuki H, Watanabe Y, Sueyoshi K, et al. (2014) Evaluation of the Time to Terminate Continuous Renal Replacement Therapy in Patients with Acute Kidney Injury Following Cardiovascular Surgery in Association with or without a Mechanical Ventilation Support. J Nephrol Ther 5: 188. doi:10.4172/2161-0959.1000188

Page 3 of 4

\section{Re-introduction of CRRT}

There were $15(47 \%)$ patients in group A and $5(12 \%)$ in group B who were re-introduced to CRRT. $(\mathrm{P}<0.001)$. (Figure 1$)$.

Adjusted odds ratio after multiple regression analysis for reintroduction of patients free from a mechanical ventilator was 5.20 (95\% confidential interval:1.15-23.4: patients with support of a mechanical ventilator as a control).

\section{Discussion}

In the present study, the incidence of patients re-introduced to CRRT was higher in those who were, rather than not supported by a mechanical ventilator when CRRT was terminated. Previously, Uchino et al. [9] proposed that urine output at the time of initial cessation of CRRT was the most important predictor of successful cessation, especially if it occurred without the administration of diuretics. In the present study, patients who were supported by a mechanical ventilator had larger urine output and higher SOFA scores with severe organ damage than those patients without support. These findings suggest that patients supported by a mechanical ventilator suffered more severe conditions or were on the way to recovery from damage of AKI after cardiac surgery. This recalls a previous study that claimed urine volume is a simple and early prognostic index for AKI patients requiring dialysis [15]. In ICU patients, the most important risk factors for AKI or mortality from AKI are often present on admission. During the ICU stay, other organ failures (especially cardiovascular) are important risk factors. Instead of urine volume, oliguric AKI was an independent risk factor for ICU mortality, and infection increased the contribution to mortality by other factors. The severity of circulatory shock is known to be the most important factor influencing outcome in AKI patients [16]. This might be related to the use of diuretics, since Van der Voort et al. [17] demonstrated that continuous infusion of furosemide in the recovery phase of hemofiltration-dependent AKI increased urine volume but did not lead to a shorter duration of renal failure. Instead of urine volume, SOFA score may be associated with organ damage. Wu et al. [10] demonstrated that less urine output, longer duration of dialysis, age over 65 years, and higher disease severity score were predictive of a patient's redialysis after initial weaning from acute dialysis. In the present study, organ damage assessed by SOFA score was higher

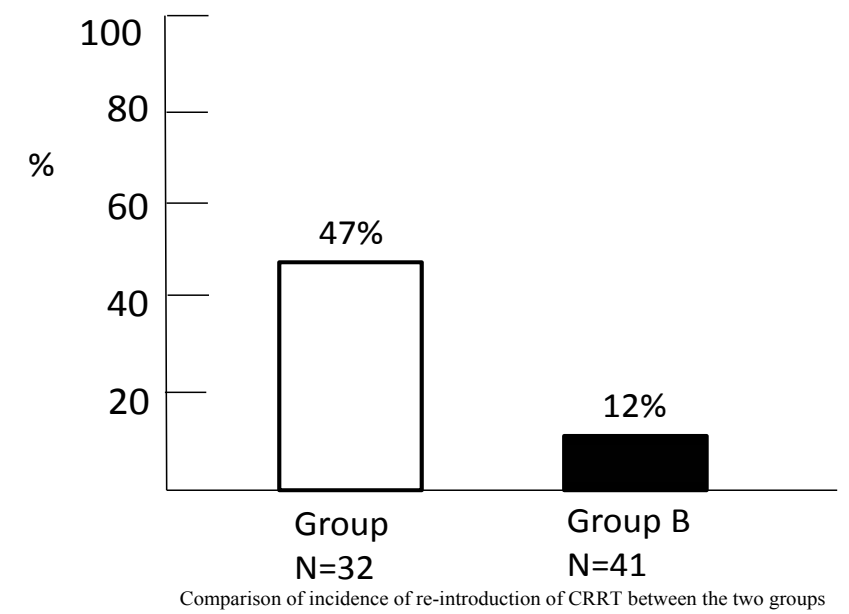

Figure 1: Comparison of incidence of re-introduction to CRRT. In patients who were weaned from a mechanical ventilator (group B), the incidence of re-introduction to CRRT was lower $(P<0.001)$ than in patients who had been supported with a mechanical ventilator until the cessation of CRRT (group A). in patients supported by a mechanical ventilator. This indicates that severity of AKI is not determined by renal score evaluated by urine volume and serum creatinine but that cardiopulmonary failure is an important factor for recovery of AKI in patients after cardiac surgery. In the studies by Uchino et al. and Wu et al. $[9,10]$, the causes of AKI were varied and AKI patients due to cardiac surgery were not the major group. In contrast, $100 \%$ of our study population was comprised of cardiac surgery induced AKI. The difference between the two studies and ours could result in different indicators for terminating CRRT. In the present study, presence or absence of a mechanical ventilator at the time of cessation of CRRT had a greater impact for re-dialysis of patients regardless of age, preoperative renal dysfunction, CVP and organ damage score. These data suggest that successful termination of CRRT in AKI patients after cardiac surgery is closely associated with removing the support of a mechanical ventilator.

A weakness of the present study is the data were derived from a single center. Inherent to the observational study design is the limitation that it establishes association but not causality. Nevertheless, it can improve individual patient care by allowing us to accurately identify when CRRT should be terminated in AKI patients after cardiac surgery. Second, all subjects of this study were patients who had received cardiac surgery. This background as causes of AKI is different from that of AKI due to various other reasons. However, compared with the previous studies that included various underlying patient's characteristics and different needs for dialysis, this study provides a clear cut proposal for successful weaning of CRRT in AKI patients after cardiac surgery. Third, the cessation of CRRT was not based on clearly established criteria and the experience comes from a single center, although general rules were followed.

Our data suggest that the cessation of CRRT was partially dependent on the two factors; the absence of a mechanical ventilator and the levels of eGFR in the patients who were not supported by mechanical ventilator before cardiovascular surgery were lower than those of patients who were supported. This might hold two things; one is a possibility of reoccurrence of AKI in spite of stability of hemodynamics, and another is prolongation of recovery from AKI. Clearly, patients' cardiopulmonary status at the time of withdrawal of mechanical ventilation would clear sufficient requirement for discontinuation of CRRT. In line with this assumption, it is not so difficult to determine when CRRT should be stopped without jeopardizing patients' general conditions.

In conclusion, albeit retrospective, our study is the first demonstration of the role for a mechanical ventilator in determining the time to terminate CRRT in AKI patients after cardiac surgery.

\section{Conflict of Interest}

The authors declare no conflict of interest in this study.

\section{References}

1. Chertow GM, Levy EM, Hammermeister KE, Grover F, Daley J (1998) Independent association between acute renal failure and mortality following cardiac surgery. Am J Med 104: 343-348.

2. Loef BG, Epema AH, Smilde TD, Henning RH et al (2005) Immediate postoperative renal function deterioration in cardiac surgical patients predicts in-hospital mortality and long-term survival. J Am Soc Nephrol 16: 195-200.

3. Hoste EA, Cruz DN, Davenport A, Mehta RL, Piccinni P, et al. (2008) The epidemiology of cardiac surgery-associated acute kidney injury. Int $\mathrm{J}$ Artif Organs 31: 158-165

4. Ostermann ME, Taube D, Morgan CJ, Evans TW (2000) Acute renal failure following cardiopulmonary bypass: a changing picture. Intensive Care Med 26 565-571. 
Citation: Tsukamoto I, Tsuchiya Y, Suzuki H, Watanabe Y, Sueyoshi K, et al. (2014) Evaluation of the Time to Terminate Continuous Renal Replacement Therapy in Patients with Acute Kidney Injury Following Cardiovascular Surgery in Association with or without a Mechanical Ventilation Support. J Nephrol Ther 5: 188. doi:10.4172/2161-0959.1000188

5. Loef BG, Henning RH, Navis G, Rankin AJ, van Oeveren W, et al. (2008) Changes in glomerular filtration rate after cardiac surgery with cardiopulmonary bypass in patients with mild preoperative renal dysfunction. British Journal of Anaesthesia 100: 759-64.

6. Seabra VF, Balk EM, Liangos O, Sosa MA, Cendoroglo M, et al. (2008) Timing of renal replacement therapy initiation in acute renal failure: a meta-analysis. Am J Kidney Dis 52: 272-284

7. Gibney N, Hoste E, Burdmann EA, Bunchman T, Kher V, et al. (2008) Timing of initiation and discontinuation of renal replacement therapy in $\mathrm{AKI}$ : unanswered key questions. Clin J Am Soc Nephrol 3: 876-880.

8. Garcia-Fernandez N, Perez-Valdivieso JR, Bes-Rastrollo M, et al. (2011) Timing of renal replacement therapy after cardiac surgery: a retrospective multicenter Spanish cohort study. Blood Purif 32: 104-11.

9. Uchino S, Bellomo R, Morimatsu H, Morgera S, Schetz M, et al. (2009) Discontinuation of continuous renal replacement therapy: a post hoc analysis of a prospective multicenter observational study. Crit Care Med 37: 2576-2582.

10. Wu VC, Ko WJ, Chang HW, Chen YW, Lin YF, et al. (2008) Risk factors of early redialysis after weaning from postoperative acute renal replacement therapy. Intensive Care Med 34: 101-108.

11. Maclntyre NR, Cook DJ, Ely EW, Jr., Epstein SK, et al. (2001) Evidence-based guidelines for weaning and discontinuing ventilatory support: a collective task force facilitated by the American College of Chest Physicians; the American
Association for Respiratory Care; and the American College of Critical Care Medicine. Chest Dec 120: 375S-95S.

12. Kellum JA, Bellomo R, Ronco C (2008) Definition and classification of acute kidney injury. Nephron Clin Pract 109: c182-187.

13. Vincent JL, Moreno R, Takala J, Willatts S, De Mendonca A, Bruining $H$, et al. (1996) The SOFA score to describe organ dysfunction/failure. On behalf of the Working Group on Sepsis-Related Problems of the European Society of Intensive Care Medicine. Intensive Care. Med 22: 707-10.

14. Matsuo S, Imai E, Horio M, Yasuda Y, Tomita K, et al. (2009) Revised equations for estimated GFR from serum creatinine in Japan. Am J Kidney Dis 53: 982992.

15. Mehta RL, Pascual MT, Gruta CG, Zhuang S, Chertow GM (2002) Refining predictive models in critically ill patients with acute renal failure. J Am Soc Nephrol 13: 1350-1357.

16. de Mendonça A, Vincent JL, Suter PM, Moreno R, Dearden NM, et al. (2000) Acute renal failure in the ICU: risk factors and outcome evaluated by the SOFA score. Intensive Care Med 26: 915-921.

17. van der Voort PH, Boerma EC, Koopmans M, Zandberg M, de Ruiter J, et al. (2009) Furosemide does not improve renal recovery after hemofiltration for acute renal failure in critically ill patients: a double blind randomized controlled trial. Crit Care Med. 37: 533-538. 